\title{
Biogeografía humana en la cuenca del Río Diamante: información arqueológica y perspectivas
}

\author{
Human biogeography in Diamante River basin: \\ archaeological information and perspectives.
}

VI Jornadas

Arqueológicas Cuyanas
Miguel Giardina*, Clara Otaola** y Fernando Franchetti ${ }^{* *}$

* Miguel Giardina, IANIGLA/CONICET, Grupo Vinculado San RafaelDepartamento de Antropología, Museo de Historia Natural de San Rafael. **Clara Otaola, IMHICIHU - CONICET. E-mail: claraotaola@conicet.gov.ar

***MHNSR, University of Pittsburgh. E-mail: ferfranchetti@gmail.com

\begin{abstract}
Resumen
En este trabajo se presentan los resultados iniciales de un proyecto de investigación arqueológica para la cuenca del río Diamante, provincia de Mendoza y se realiza una síntesis de la información disponible. Como objetivo general y de largo plazo, buscamos analizar si hubo una disminución en la movilidad residencial y una ampliación en la variedad de recursos consumidos durante el Holoceno tardío como plantea el modelo de poblamiento del área propuesto a nivel regional. O por el contrario, analizar si existió un continuum de sociedades con alta movilidad residencial sin una saturación del espacio que implicara cambios en el uso de los recursos. Las evidencias arqueológicas disponibles para evaluar estos modelos alternativos no son suficientes como para inclinar la balanza hacia uno u otro modelo. Sin embargo, la tendencia general observada apunta hacia una amplia movilidad residencial y el aprovechamiento de recursos de todos los pisos ecológicos.
\end{abstract}

Palabras clave: Biogeografía Humana; Río Diamante; Cazadores-Recolectores; Intensificación.

\begin{abstract}
In this paper, first results of an archaeological research project at Diamante river basin, Mendoza province are presented. The main objective is to analyze whether there were a diminution in residential mobility and an intensification in the use of resources during the late Holocene as posed by a regional model. The alternative hypothesis is that existed a continuum of economic and social lifeways that implied a high residential mobility system that would not involve changes in the use of resources. Even though there are not enough archaeological evidences yet to incline the balance to one model or the other, the general trend points to a high residential mobility and the use of resources from all the ecological floors.
\end{abstract}

Keywords: Human Biogeography; Diamante River; Hunter-Gatherers; Intensification.

El modelo biogeográfico utilizado para explicar el proceso de poblamiento y uso del espacio de poblaciones humanas en el sur de Mendoza, aborda fundamentalmente dos cuestiones; la cronología de las ocupaciones y el uso de los recursos. En relación con el primer aspecto, se ha demostrado que aquellos espacios con mayor abundancia y predictibilidad de recursos han sido ocupados primero y en segundo lugar aquellas áreas más hostiles y riesgosas (Neme y Gil 2008a). En lo que respecta a la segunda cuestión, se planteó que hacia los 2000 años A.P, hubo un aumento demográfico, una ocupación efectiva del área y una saturación del espacio que condujo, entre otras cosas, a una disminución en la movilidad residencial y al desarrollo de un proceso de intensificación (Neme 2007, Neme y Gil 2008a, 2008b). Esta reducción de la movilidad residencial, habría provocado el agotamiento de los recursos locales de mayor rendimiento y como consecuencia, se habría incorporado una mayor cantidad y variedad de alimentos de bajo rendimiento económico (Gil 2003; 2006; Neme 2007; Neme y Gil 2008a; 2008b Neme et al. 2015).

Si bien existen algunas evidencias que apoyan este modelo de intensificación (Neme 2007; Llano 2012), en trabajos posteriores se ha señalado que el registro arqueológico del área no siempre es concordante con el mismo (Neme et al. 2015; Otaola et al. 2015). Investigaciones a nivel regional sobre el consumo de animales (incluyendo registro arqueológico de las cuencas del río Diamante, río Atuel y río Grande, al sur de la provincia de Mendoza), 
señalan que la principal causa de variabilidad en los conjuntos zooarqueológicos no han sido producto del proceso de intensificación sino al uso de la fauna local de los distintos ambientes a medida que estos son ocupados, señalando que existe una predominancia de consumo de recursos locales en cada área (Corbat 2016; Giardina 2010; Neme et al. 2015; Otaola 2013; Otaola et al. 2015). En un modelo alternativo que aún no ha sido completamente testeado, se plantea la posibilidad de que el aumento demográfico no se habría dado acompañado de una saturación del espacio y una reducción de la movilidad residencial, sino que por el contrario habría habido un aumento en la movilidad residencial, con un mayor rango de acción en el que se incluyen áreas y recursos antes no utilizados (Borrero 2012).

A partir de las evidencias arqueológicas reunidas en la cuenca del río Diamante, analizaremos si el registro arqueológico se comporta de acuerdo a algunas de las expectativas planteadas en los modelos mencionados. Si bien la información disponible en la actualidad no permite realizar un análisis acabado de cuál de estos dos modelos sería el más parsimonioso, el objetivo específico de este trabajo es presentar la información arqueológica de la cuenca del río Diamante reunida hasta el momento y plantear las expectativas de dichos modelos para testear en trabajos futuros. El objetivo general es entender el uso del espacio y de los recursos por parte de las poblaciones que ocuparon la mencionada cuenca, teniendo en cuenta fundamentalmente la relación entre las variables geográficas, ambientales y climáticas en el desarrollo de las estrategias de subsistencia. Para ello es necesario incluir los resultados de los trabajos previos y considerar las características ambientales del área.

\section{Antecedentes en el área del río Diamante y alrededores}

Existe una gran cantidad de información arqueológica generada previamente por distintos investigadores, la cual ha sido escasamente publicada. Las primeras menciones sobre hallazgos arqueológicos recuperados sin una metodología sistemática las encontramos en el trabajo de Sosa Morales (1979), quien realizó una síntesis de sitios arqueológicos en las proximidades de la Villa 25 de Mayo, El Médano de Los Tolditos, Agua de los Cielos, La Cancha entre otros. Para Los Tolditos describe sitios arqueológicos a cielo abierto como así también la presencia de un alero rocoso. Rusconi (1962) hace mención de varias localidades arqueológicas en las inmediaciones del río Diamante en sus "jiras" por el sur de Mendoza. Divide esta zona en dos; por un lado los sitios que encuentra al oeste de la estancia Los Reyunos, actualmente la cabecera del distrito de la Villa 25 de Mayo, describiendo sitios en reparos rocosos con arte rupestre, sitios a cielo abierto en médanos, muchos de ellos con presencia de restos humanos, instrumentos líticos, elementos cerámicos alóctonos y restos de animales. Otro de los investigadores pioneros del área fue Gambier. Excepto por breves menciones en algunos de sus trabajos (Gambier 1979; 1985) y por el estudio de las colecciones arqueobotánicas (Andreoni 2014; Llano 2012; Llano et al. 2012), los materiales recuperados en sitios excavados por Gambier en esta zona nunca fueron dados a conocer en su totalidad.

Los trabajos de Lagiglia (1977) en el área comprendida entre la localidad de Villa 25 de Mayo y el cerro del Diamante le dieron un enfoque histórico cultural al registro arqueológico de esa región. A partir de los hallazgos líticos en los niveles aterrazados de la Formación Colonia los Coroneles (Sepúlveda et al. 2001), realizó una caracterización de las sociedades de cazadores recolectores que habitaron la región que denominó "Cultura de los Coroneles". Para Lagiglia, esta "cultura" se dividía en tres estadios cronológicos y evolutivos (Coroneles I, II y III), transitando desde una etapa en la que sólo se confeccionaban lascas unifaciales de gran tamaño (Los Coroneles I), hasta la elaboración de puntas bifaciales foliáceas pequeñas, raederas y raspadores (Los Coroneles III) de tamaño más reducido que en estadios anteriores. A esta caracterización cultural, Lagiglia le atribuye una antigüedad de entre 10000 años A.P. y 5000 años A.P. a partir de correlaciones de los estudios geocronológicos que realiza Polanski (1963).

Entre los trabajos más recientes realizados en esta cuenca se destacan las excavaciones realizadas por Durán y colaboradores en la Laguna del Diamante, localizada en las nacientes del río homónimo (Figura 1) (Durán et al. 2006). En esta laguna se han registrado 15 sitios compuestos cada uno por un número variable de estructuras pircadas, y dos fuentes secundarias de obsidiana (Cortegoso et al. 2014, Cortegoso et al. 2016). Por otro lado, las recientes excavaciones en el sitio Risco de los Indios, también localizado en la cuenca superior del Diamante, brindan información de utilidad para completar la caracterización de estos sitios localizados en la alta Cordillera (Neme et al. 2016).

Las metodologías implementadas para abordar las preguntas de investigación en cada caso fueron diversas y sin un objetivo en común. En este trabajo utilizaremos la información disponible gracias a estas investigaciones previas así como información cronológica y zooarqueológica generada por nosotros en los últimos años.

\section{Información ambiental}

De acuerdo con los aspectos teóricos del modelo biogeográfico de Neme y Gil (2008a) en el que se evalúa la relación entre variables geográficas, ambientales y climáticas en las estrategias humanas, resulta fundamental realizar una jerarquización del espacio de acuerdo con la disponibilidad de recursos existentes y 
conocidos hasta el momento. Esta jerarquización puede realizarse considerando la biodiversidad disponible en cada una de las tres provincias fitogeográficas atravesadas por la cuenca del río Diamante: se trata de las provincias fitogeográficas Altoandina, Patagonia y Monte (Cabrera 1976; Hernández 2002; Mares et al. 1985), también denominadas en nuestro trabajos como desierto Andino, Patagónico y de Monte (Roig 1972) (Figura 1). Cada uno de estos desiertos posee características particulares que imprimen variabilidad en la disponibilidad de recursos aprovechables para las sociedades de cazadores recolectores que habitaron esta región.

Aunque no haya una estricta correlación entre altitud y diversidad (Mc Cain y Grytnes 2010; Rahbek 1995), existe un patrón de disminución de la abundancia y diversidad de flora y fauna a medida que aumenta la altitud (Pianka 1982). Esto ha sido caracterizado en los estudios zoológicos y botánicos en la provincia de Mendoza (Roig 1972; Roig et al. 2000; Videla et al. 1997). El desierto Andino se desarrolla en un clima de alta montaña, frío y seco con heladas todo el año y precipitaciones en forma de nieve. La vegetación es pobre, formada principalmente por estepas graminosas o de caméfitos en cojín, compuestos por especies de Mulinum sp. Crassifolium sp. y Adesmia subterranea (Cabrera 1971; Roig 1972). Se registra también la presencia de vegas, en las que dominan juncáceas y ciperáceas (Roig 1972). En cuanto a la fauna disponible en este desierto, se halla aquí la "fauna de montañas" (Roig 1972), entre los 2300 msnm y hasta donde la vegetación desaparece (4000 msnm). Esta fauna se concentra en los espacios en los que hay pastizales o coironales. Se encuentran aquí mamíferos de tamaño grande como el puma (Felis concolor), el zorro colorado (Pseudalopex culpaeus) y el guanaco (Lama guanicoe), mamíferos de tamaño pequeño como los roedores Akodon andinus, Ctenomys mendocinus y Phyllotis darwini y aves de familias como Thinocoridae, Anatidae en áreas de vegas y también algunos paseriformes.

El desierto Patagónico se desarrolla entre los 2500 y 1000 msnm (Figura 1). Se caracteriza por tener un clima seco y frío, con vientos intensos. La vegetación que predomina es de gramíneas, dicotiledóneas, estepas arbustivas y en las partes de mayor humedad estepas graminosas. La presencia de algunos elementos de monte, imprimen el carácter de ecotono entre el Monte y Patagonia (Cabrera 1971). En cuanto a la fauna, por debajo de los 2500 msnm, el guanaco y el puma siguen siendo los mamíferos de mayor porte, pero el zorro colorado es aquí reemplazado por el zorro gris (Pseudalopex griseus). Otros mamíferos carnívoros son el gato montés (Felis geoffroyi) y el zorrino (Conepatus chinga). Se hallan también aquí roedores como el chinchillón (Lagidium viscacia), la mara (Dolichotis patagonum) y otros de tamaño pequeño (peso corporal menor a $1 \mathrm{~kg}$ ). Hay escasa presencia de edentados pero pueden hallarse aquí especímenes de peludo (Chaetophractus villosus) y piche (Zaediyus pichiy). Además se observa una gran cantidad de aves, entre las que se destacan choique (Rhea pennata), Anátidos, aves rapaces, columbiformes y paseriformes. También es posible encontrar saurios (batracios y reptiles) y peces (Pygidium borelli y Hatcheria sp.) (Roig 1972).

Finalmente, el desierto de Monte se desarrolla entre los 1000 y 300 msnm, y se extiende desde el comienzo del bloque de San Rafael en el extremo NO, hasta el río Desaguadero, en el borde este de la provincia de Mendoza (Figura 1). La complejidad de la biota probablemente se debe al extensivo contacto entre dos tipos de biotas de Sudamérica: Biota tropical y Biota patagónica (Roig et al. 2009). Las precipitaciones varían entre 80 y 250 $\mathrm{mm}$ anuales y las temperaturas medias anuales oscilan entre los $13^{\circ} \mathrm{C}$ y $17.5^{\circ} \mathrm{C}$. Dos tipos de comunidades vegetales predominan en este área, la más comunes son las Jarillas (Larrea divaricata, L. cuneifolia y L. nitida), que forman arbustos de follaje perenne de 1,5 a $3 \mathrm{~m}$ de altura (Roig 1972). Las otras plantas características del monte son los algarrobos Prosopis flexuosa y P. chilensis, pero también se encuentran arbustos leguminosos o árboles que alcanzan 8 a 10 m de altura (Roig1972; Roig et al., 2000). La fauna característica del monte está integrada por edentados, gatos salvajes y puma. Los roedores son también abundantes, y se registran de diversos tamaños, tales como mara (Dolichotis patagonum), chinchilla (Lagidium vizcacia) y vizcacha (Lagostomus maximus) y ratones de menor tamaño como Microcavia australis, Galea lucoblephara, Akodon andinus, Graomys griseflavus entre otros. En cuanto a las aves, encontramos aquí de porte variado, tales como ñandú (Rhea americana), perdices (Eudromia elegans), falconiformes, columbiformes y paseriformes. También existe un abundante número de anfibios y reptiles, entre los que se pueden mencionar, anuros, ofidios, lagartijas (Liolaemus sp.) y tortugas (Chelonoidis chilensis) (Roig 1972).

\section{Expectativas para el poblamiento de la cuenca del rio Diamante, la movilidad humana y el consumo de recursos.}

Desde una perspectiva de la ecología evolutiva (Pianka 1983) y en relación con los modelos de predación óptima aplicados a la antropología (Bettinger 1991; Kelly 1995; Bird y O'Connell 2006; Shennan 2002; Winterhalder y Smith 1992), se espera que la ocupación de cada uno de los ambientes descriptos esté relacionada con los costos de obtención de recursos. En este sentido, siguiendo el modelo generado por Neme y Gil (2008a) para la región, se espera también en la cuenca del Diamante que los ambientes con mayor productividad primaria y accesibilidad sean ocupados primero. Siguiendo el orden de poblamiento propuesto, esperamos que las provincias fitogeográfica de Patagonia y de Monte hayan sido habitadas primero. Por otro lado, se espera que la 


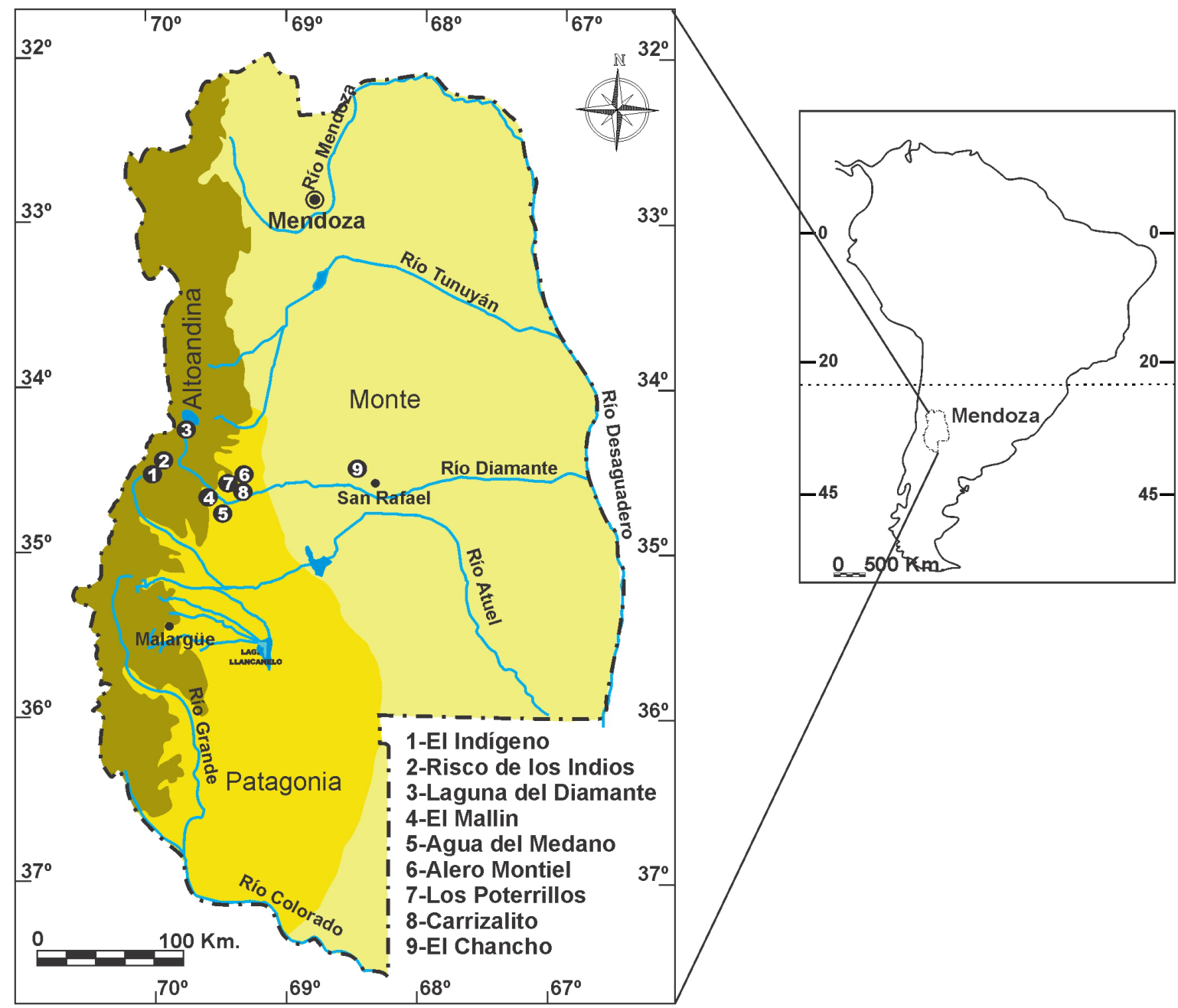

Figura 1. Provincias fitogeográficas y sitios arqueológicos mencionados en este artículo

Figure 1. Phytogeographic provinces and archaeological sites mentioned in this article.

provincia Altoandina haya sido ocupada recién hacia el Holoceno tardío, únicamente cuando las condiciones demográficas hicieron necesario utilizar espacios estacionales y de menor productividad primaria (Borrero 1989-1990, Neme y Gil 2008a).

En cuanto al aspecto vinculado a la obtención y consumo de recursos, si se sigue el modelo de intensificación planteado por Neme y Gil (2008a y b) se espera que haya una disminución en los recursos de menor rendimiento económico y un aumento en la diversidad de taxa explotada en la segunda mitad del Holoceno tardío. Sin embargo, si consideramos el modelo alternativo, se espera que por el contrario, exista cierta continuidad en el tipo de recursos explotados y que en cada área se registre una explotación de recursos acorde a la oferta de los mismo.

A continuación presentaremos la información arqueológica generada hasta el momento para poder evaluar si se cumplen algunas las expectativas de alguno de estos dos modelos alternativos.

\section{El registro arqueológico}

\section{Desierto Andino}

Los sitios arqueológicos en el desierto Andino son, Laguna del Diamante, Risco de los Indios y El Indígeno (Figura 1). Estos sitios presentan características comunes y distintivas en relación al resto de los sitios arqueológicos de la región. Poseen una cronología correspondiente a la segunda mitad del Holoceno tardío (Figura 2) y su rasgo principal es la presencia de grupos de estructuras habitacionales semicirculares construidas con muros pircados, utilizadas como lugares de vivienda (Durán et al. 2006; Neme 2002; 2007; Neme et al. 2016). Presentan una mayor cantidad de cerámica en comparación con otros sitios del sur de Mendoza y una fuerte presencia de materiales alóctonos, lo que constituye un fuerte indicio de contacto entre poblaciones de otras regiones.

Otras características que comparten son las evidencias de consumo de fauna local, que incluye principalmente 
al guanaco y avifauna en los sitios localizados en vegas y lagunas. Estos recursos además son complementados con otros no locales, de áreas más bajas como los dasipódidos, huevos de reídos y plantas domésticas (Durán et al. 2006; Neme et al. 2016, Tabla 1). El registro zooarqueológico presenta muy mala preservación lo que dificulta la identificación a nivel específico de los especímenes óseos, siendo los estadios de meteorización elevados como así también el grado de fragmentación de los mismos, lo que genera porcentajes altos de especímenes indeterminados tanto a nivel taxonómico como anatómicos. Existen también evidencias de consumo de plantas silvestres y en menor medida, de plantas domesticadas. En el sitio Laguna del Diamante (LD-4) se hallaron unos 20 restos arqueobotánicos, de especies domésticas. Se estima que fueron introducidos recientemente en el sitio, ya que se encontraron en los niveles superiores de la excavación, asociados a otros materiales como vidrio y monedas (Durán et al. 2006). En cuanto al único taxón silvestre hallado, Maihueniopsis sp. los investigadores señalan que es una cactácea que se desarrolla en el piedemonte y valles orientales, por lo que habría sido transportado desde la vertiente oriental (Durán et al. 2006) (Tabla 2). En el sitio El Indígeno se encontraron restos de Zea mays y Lagenaria siceraria (epicarpio), plantas domesticas cultivadas en pisos altitudinales inferiores al de este sitio, y Empetrum rubrum, una planta silvestre que crece en zonas alpinas (Neme 2016). En el sitio Risco de los Indios, los restos de los taxones silvestres recuperados (Schinus johnstonii, Maihuenia patagónica, Maihueniopsis glomerata y Anarthrophyllum rigidum) crecen en la actualidad dentro de la provincia fitogeográfica Altoandina (Böcher et al. 1972; Muiño et al. 2012; Roig 1972), lo que hace suponer que los mismos debieron ser colectados en las proximidades del sitio. Para dichos sitios solamente se ha recuperado Phaseolus vulgaris como taxa domesticada, el mismo está bien documentado en Chile central (Planella y Tagle 2004), y se han identificado especímenes en la vertiente oriental de la cordillera, especialmente en la planicie a más de $150 \mathrm{~km}$ de distancia del sitio (Lagiglia 1968; Llano y Durán 2014).

En relación a la tecnología lítica, existen diferencias en la representación de materias primas en estos sitios de altura. Mientras que en LD-4 predominan los artefactos de obsidiana en porcentajes mayores al 70\%, en Risco de los Indios hay mayor abundancia de basaltos (46\%), siguiendo en orden de importancia las rocas silíceas (21\%), las obsidianas (19\%), la arenisca silicificada (7\%) y por último las riolitas (4\%). Respecto a la procedencia de obsidiana, si bien Risco de los Indios se encuentra a $30 \mathrm{~km}$ lineales de la fuente Laguna del Diamante, los análisis de elementos traza realizados sobre cinco artefactos indican que los mismos provienen de dos fuentes no locales: Las Cargas, localizada a ca. $100 \mathrm{~km}$ de distancia y Coche quemado, localizada a $200 \mathrm{~km}$ lineales. En LD-4, un gran porcentaje de las obsidianas $(89,6 \%)$ provienen de las fuentes locales de la Laguna Diamante que se encuentran a menos de 10 km del sitio (Cortegoso et al. 2014; Durán et al. 2012). La presencia de estas fuentes cercanas explica la predominancia de esta materia prima en este sitio. Por otro lado, aunque se encuentren representadas en un porcentaje menor, se registró la presencia de obsidianas provenientes de Las Cargas (distante a $100 \mathrm{~km}$ ) y Laguna Negra-Maule (220 km) (Cortegoso et al. 2014). En cuanto al sitio El Indígeno, los análisis de obsidianas señalan que las mismas provienen de Las Cargas localizadas ca. 90 $\mathrm{km}$ hacia el sur de este sitio y dos muestras pertenecen a una fuente cuya localización aún se desconoce (Durán et al. 2004; Neme 2016).

La cerámica hallada en estos sitios se origina en ambas vertientes cordilleranas; en el caso de LD-4 se hallaron tiestos característicos de los denominados Período Agroalfarero Temprano, Intermedio Tardío y Tardío de Chile. Se trata de cerámica decorada, de tipología Aconcagua e Inca de Chile Central, aunque también se registraron otros estilos característicos del Norte Chico que también se observan en sitios del noroeste de San Juan. También se han hallado tiestos de cerámica no decorada a lo largo de toda la secuencia (Durán et al. 2006). En el caso de Risco de los Indios, todos los tiestos decorados corresponden a estilos no locales, provenientes de la vertiente occidental de la cordillera (Aconcagua y Diaguita). Los tiestos no decorados predominan en la muestra (94\%) y corresponden al estilo Overo/Nihuil, de la vertiente oriental (Neme et al. 2016).

\section{Desierto Patagónico}

Algunos de los sitios localizados en el Desierto Patagónico han sido excavados en la década de 1970, tal es el caso de los sitios El Carrizalito, Los Potrerillos, El Mallín y Alero Montiel (Gambier 1979; 1985). Por otro lado, el sitio Agua del Médano (Figura 1), fue registrado por primera vez en la década de 1940, pero el mismo fue excavado recientemente. Excepto por este último, los restantes sitios se encuentran localizados en cuevas. En conjunto, la cronología de estos sitios comprende fines del Holoceno temprano, una ausencia de ocupaciones durante el Holoceno medio (específicamente entre los 8200 y 3600 años A.P) y evidencias de ocupaciones hacia fines del Holoceno tardío (Figura 2).

La fauna recuperada en los sitios excavados por Gambier puede haber sufrido sesgos a causa de los métodos de excavación y de los objetivos de investigación perseguidos. En Agua del Médano, que ha sido excavado por nosotros, las malas condiciones para la preservación que presentan los restos pueden haber sesgado tafonómicamente la muestra. A pesar de estas dificultades, la evidencia disponible muestra diferencias en cuanto a los taxones representados respecto a los sitios del desierto Andino (Tabla 1). A excepción de los camélidos que se encuentran en los sitios de ambos pisos altitudinales, en este sector de la cuenca se observa cáscara de huevos de Rheidae, 


\begin{tabular}{|c|c|c|c|c|c|c|}
\hline \multirow{3}{*}{$\begin{array}{l}\text { Zooarqueología } \\
\text { Taxón/Sitio }\end{array}$} & \multicolumn{3}{|c|}{ Desierto Altoandino } & \multicolumn{3}{|c|}{ Desierto Patagónico } \\
\hline & El Indígeno & Laguna & Risco de los & Alero Montiel & Agua del & Carrizalito \\
\hline & & Diamante 4 & Indios & & Médano & \\
\hline Fauna Indet & 151 & 14539 & 1088 & 48 & 218 & 30 \\
\hline Ave indet & 2 & 27 & - & 4 & - & 8 \\
\hline Charadriiformes & - & 2 & - & - & - & - \\
\hline Anseriformes & - & 1 & - & - & - & - \\
\hline Paseriforme Indet. & - & 1 & - & - & - & - \\
\hline Suboscine & - & 1 & - & - & - & - \\
\hline Anas sp. & - & 6 & - & - & - & - \\
\hline Anas platalea & - & 1 & - & - & - & - \\
\hline Anas specularioides & - & 2 & - & - & - & - \\
\hline Attagis gayi & - & 1 & - & - & - & - \\
\hline Choelephaga melanoptera & - & 1 & - & - & - & - \\
\hline Rheidae & - & $1 *$ & - & $611^{*}$ & $9 *$ & $139 *$ \\
\hline Rhea pennata & - & - & - & - & - & 1 \\
\hline Mamiferos indet & 10 & 320 & 255 & 50 & 16 & 18 \\
\hline Equs caballus & - & - & - & - & - & 1 \\
\hline Artiodactyla & 9 & 24 & - & 4 & - & 1 \\
\hline Camelidae & 9 & 174 & 35 & 65 & 6 & 12 \\
\hline Lama guanicoe & 23 & 83 & 49 & 37 & 1 & 3 \\
\hline Caprininae & - & 17 & - & - & - & - \\
\hline Felis concolor & - & - & - & 1 & - & 2 \\
\hline Pseudalopex culpaeus & - & - & 1 & - & - & - \\
\hline Pseudalopex griseus & - & - & - & 1 & - & - \\
\hline Lagostomus maximus & - & - & - & 1 & - & - \\
\hline Lagidium viscacia & - & - & - & 7 & - & - \\
\hline Ctenomys sp. & - & - & - & 5 & 1 & 2 \\
\hline Sigmodontidae & - & - & - & - & 2 & - \\
\hline Dasipodidae & - & 10 & 3 & $4(13)$ & $19(18)$ & $4(17)$ \\
\hline Chaetophractus sp. & - & - & - & $2(3)$ & - & - \\
\hline Zaedyus sp. & - & 3 & - & 5 & - & 4 \\
\hline Iguanidae & - & - & - & 1 & 1 & - \\
\hline Mollusca & - & - & - & $1^{* *}$ & - & 3 \\
\hline Serpentes & - & - & - & - & - & 1 \\
\hline NTAXA & 2 & 10 & 3 & 7 & 2 & 3 \\
\hline Índice de Diversidad (Shannon) & 0,1 & 0,27 & 0,21 & 0,75 & 0,3 & 0,66 \\
\hline
\end{tabular}

Tabla 1. Registro de fauna con evidencias de consumo en sitios de la cuenca del río Diamante. *Fragmentos de cáscara de huevo de Rheidae. ** Cuentas de collar. Los datos de El Indígeno fueron tomados de Neme 2007, los de Laguna Diamante 4 de Durán et al. 2006 y Giardina 2010 y los de Risco de los Indios fueron publicados en Neme et al. 2016.

Table 1. Archaeofaunal record with evidence of human consumption. * Fragments of eggshell Rheidae. ** Necklacebeads. Data from El Indígeno was extracted from Neme 2007; Laguna Diamante 4 from Durán et al. 2006 and Giardina 2010; Risco de los Indios faunal data had been published in Neme et al. 2016. 


\begin{tabular}{|c|c|c|c|c|c|c|}
\hline \multirow{2}{*}{$\begin{array}{l}\text { Arqueobotánica } \\
\text { Taxón/Sitio }\end{array}$} & \multicolumn{3}{|c|}{ Desierto Altoandino } & \multicolumn{3}{|c|}{ Desierto Patagonia } \\
\hline & $\begin{array}{c}\text { El } \\
\text { Indígeno }\end{array}$ & $\begin{array}{c}\text { Laguna } \\
\text { Diamante } 4\end{array}$ & $\begin{array}{l}\text { Risco de los } \\
\text { Indios }\end{array}$ & $\begin{array}{l}\text { Alero } \\
\text { Montiel }\end{array}$ & El Mallín & El Carrizalito \\
\hline Zea mays & 5 & - & - & $4^{*}$ & - & 45 \\
\hline Schinus polygamus & - & - & - & 1 & - & 649 \\
\hline Propospis alpataco & - & - & - & $1^{* *}$ & - & - \\
\hline Cucurbitacea & - & 1 & - & - & - & - \\
\hline Lagenaria $\mathrm{sp}$ & 1 & - & - & - & 1 & 1 \\
\hline Phragmites australis & - & - & - & - & - & 1 \\
\hline Solanum elaegniflium & - & - & - & - & - & 2 \\
\hline Empetrum rubrum & 2 & - & - & - & - & - \\
\hline Chusque culeou & - & - & - & - & 5 & 1 \\
\hline Maihuniopsis sp. & - & 5 & - & - & - & - \\
\hline Maihuniopsis darwinii & - & - & - & - & - & 1 \\
\hline Anarthrophyllum rigidum & - & - & 7 & - & - & - \\
\hline Maihuenia patagonica & - & - & 3 & - & - & - \\
\hline Maihueniopsis glomerata & - & - & 1 & - & - & - \\
\hline Phaseolus vulgaris & - & - & 22 & - & - & - \\
\hline Schinus johnstonii & - & - & 5 & - & - & - \\
\hline Diplolepis hieronymi & - & - & - & - & 6 & - \\
\hline Opuntia sulphurea & - & - & - & - & 1 & - \\
\hline NTAXA & 3 & 2 & 5 & 1 & 4 & 6 \\
\hline $\begin{array}{l}\text { Índice de Diversidad (Shannon } \\
\text { W) }\end{array}$ & 0,9 & 0,4 & 1,1 & 0 & 1,1 & 0,15 \\
\hline
\end{tabular}

Tabla 2. Información arqueobotánica. *Framgentos de marlo menores a $1 \mathrm{~cm}^{2},{ }^{* *} / n s t r u m e n t o . ~ P u n t a$ ahusada, termoalterada. Los datos arqueobotánicos fueron tomados de Durán et al. 2006:116; Llano et al 2012:6; Neme 2016 y Neme et al. 2016.

Table 2. Archaeobotany information. ${ }^{* * *}$ Fragments of less than $1 \mathrm{~cm}^{2 * * * *}$ instrument. tapered tip, carbonization. Archaeobotanical data were taken from Durán et al. 2006: 116; Llano et al 2012: 6; Neme 2016, Neme et al. 2016.

roedores de tamaño mediano, como chinchillón, vizcacha y peludos. Otro animal de gran porte que se ha encontrado en estos sitios es el puma que a pesar de que su distribución corresponde también a la provincia Altoandina (Tabla 1).

Los restos arqueobotánicos recuperados son escasos en todos los sitios excepto en El Carrizalito, mientras que en Agua del Médano no se halló este tipo de evidencia. En Alero Montiel sólo se recuperaron seis macrorrestos vegetales, de los cuales cuatro corresponden a pequeños fragmentos de marlo de maíz de tamaño menor a $1 \mathrm{~cm}^{2}, y$ los otros dos restantes corresponden a una punta ahusada y termoalterada, manufacturada sobre la porción leñosa de Prosopis alpataco, y a un endocarpo de Schinus Polygamus. El Carrizalito presenta un mayor número de restos arqueobotánicos en donde predominan restos de la planta silvestre Schinus polygamus y sigue en orden de importancia granos de Zea mays (Llano et al. 2012) (Tabla 2). En menor medida hay restos de otras plantas silvestres, entre las que se destaca la presencia de Chusquea culeou proveniente de la vertiente occidental del pacífico (Llano 


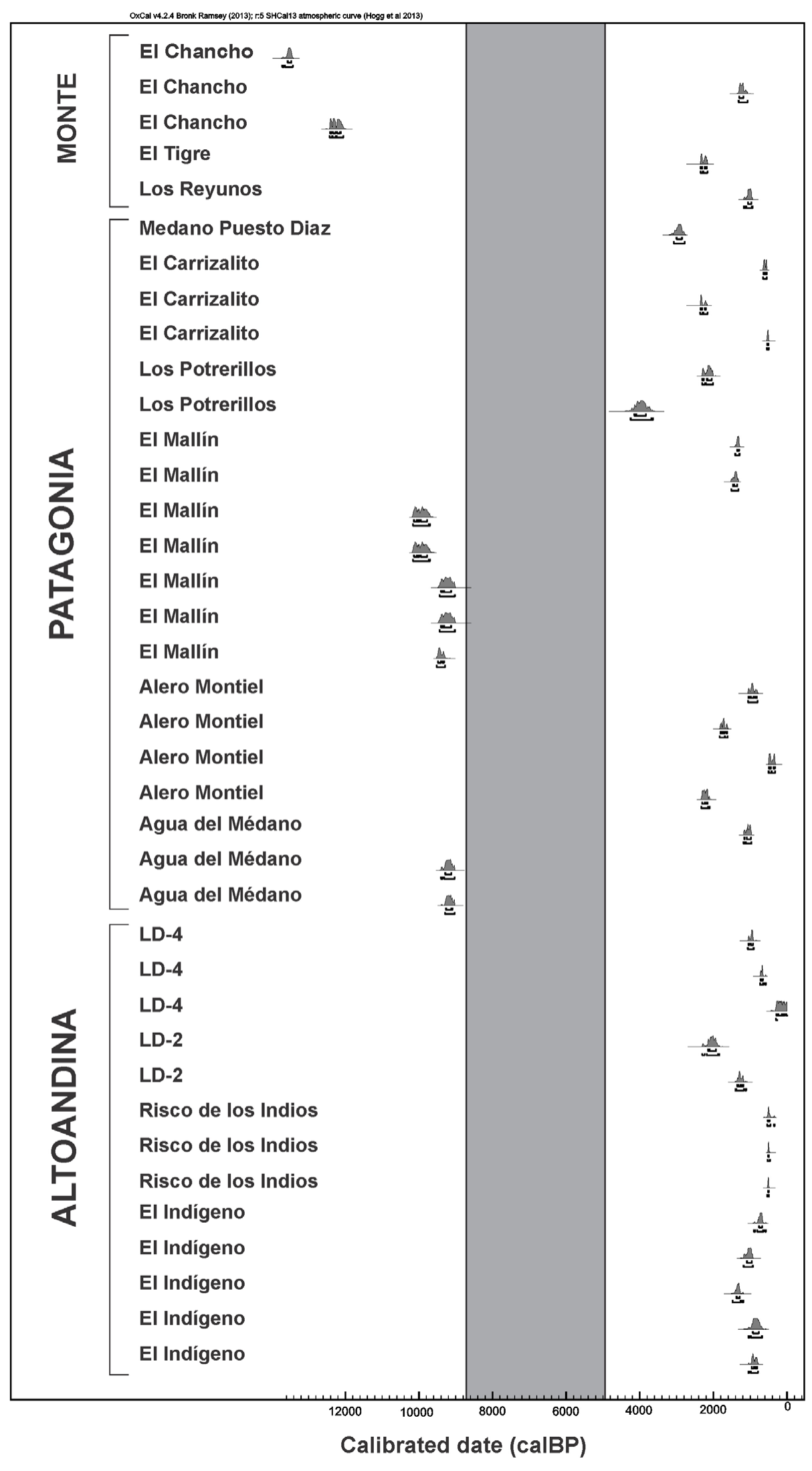

Figura 2. Fechados radiocarbónicos calibrados (Oxcal, ShCal 13) de la cuenca del río Diamante. Área gris señala la ausencia de fechados durante el Holoceno medio. Las información radiocarbónica proviene de los siguientes artículos: Durán et al. 2006 (LD-4 y LD-2); Gil et al. 2009 (El Tigre, Médano Puesto Díaz, Los Reyunos); Llano et al. 2012 (A. Montiel, Carrizalito, Potrerillos), Neme 2007 (El indígena); Neme et al. 2016 (Risco de los Indios); Tripaldi et al. 2009 (El Chancho).

Figure 2. Calibrated radiocarbon dates. Grey area shows the chronological gap during the Middle Holocene. Radiocarbon data extracted from Durán et al. 2006 (LD-4 y LD-2); Gil et al. 2009 (El Tigre, Médano Puesto Díaz, Los Reyunos); Llano et al. 2012 (A. Montiel, Carrizalito, Potrerillos), Neme 2007 (El indígena); Neme et al. 2016 (Risco de los Indios); Tripaldi et al. 2009 (El Chancho).

a consumir vegetales silvestres de menor rendimiento. Por otro lado, tal como ha sido señalado en trabajos macroregionales a partir de las evidencias arqueobotánicas e isotópicas las plantas domésticas no habrían constituido un recurso base en la dieta de las poblaciones que habitaron estos sitios (Llano et al. 2012, Gil et al. 2011).

En cuanto a la tecnología lítica y cerámica, por el momento solo disponemos de información proveniente del sitio Agua del Médano y de análisis geoquímicos de obsidianas recuperadas en el resto de los sitios. Las obsidianas analizadas en los sitios de la provincia Patagónica, provienen de canteras localizadas en algunos casos a más de 170 km en línea recta como es el caso de las obsidianas del Maule halladas en el sitio El Mallín en donde también se encontró obsidiana proveniente de la cantera Las Cargas, distante a unos 100 km et al. 2012). Por último, no se han documentado restos arqueobotánicos del sitio Los Potrerillos. Llano, (Llano et al. 2012), señala que las plantas domésticas habrían sido incorporadas a la dieta únicamente cuando los recursos de mayor rendimiento económico decrecieron y se comenzó en línea recta (Durán et al. 2012; Giesso et al. 2011). En el sitio Los Potrerillos, se hallaron obsidianas de la fuente Las Cargas (ca. 100 km), Laguna el Diamante Arroyo Paramillos (a $40 \mathrm{~km}$ aproximadamente) y una fuente cuya localización es aún desconocida. En el sitio El Carrizalito se 
hallaron obsidianas provenientes de Las Cargas, localizada a unos $120 \mathrm{~km}$ de este sitio. Las obsidianas de Agua del Médano provienen de una fuente desconocida y de la cantera extra cordillerana El Peceño, localizada a $100 \mathrm{~km}$ en dirección sureste.

En Agua del Médano, se han recuperados apenas dos tiestos de cerámica incisa que ha sido caracterizada como de estilo Agrelo, y 174 artefactos líticos, de los cuales únicamente tres son instrumentos y el resto desechos de talla que corresponde a estadios finales de formatización y mantenimiento de filos. Dentro de las materias primas presentes, predominan los basaltos (96\%), y están presentes también sílices (2\%), Obsidiana (1\%) y otras materias primas no identificadas.

\section{Desierto de Monte}

En el desierto de Monte contamos con información proveniente de fechados de esqueletos humanos aislados, como en el caso de los esqueletos hallados en Los Reyunos y Arroyo el Tigre que datan del Holoceno tardío e información proveniente del sitio El Chancho, con dataciones del Pleistoceno final, Holoceno temprano y Holoceno tardío (Neme et al. 2009, Tripaldi et al. 2009) (Figura 2). El Chancho es un sitio de baja resolución temporal en el que los materiales fechados se presentan en un depósito de $10 \mathrm{~cm}$ de espesor. La deflación de los depósitos eólicos que cubre la planicie de agradación donde se encuentran los materiales arqueológicos pudo haber sido la causa principal para que se produzca el palimpsesto (Tripaldi et al. 2009). Los materiales arqueológicos incluyen unos pocos especímenes óseos, artefactos líticos confeccionados en rocas locales provenientes del cauce del zanjón Agua del Chancho. El conjunto lítico está compuesto por 248 artefactos, entre los que se observan solo dos instrumentos (una punta de proyectil de sílice y una lasca con microretoque sumario), dos núcleos, y el resto corresponde a desechos de talla. La materia prima más representada es la cuarcita (41\%), seguida por el sílice (28\%), basalto e indiferenciada $(10 \%)$, arenisca $(4 \%)$, arenisca cuarcítica $(3 \%)$, andesita (2\%) y finalmente la calcedonia y porfiro (1\%). En dicho sitio no se observa la presencia de obsidiana (Neme et al. 2009). En cuanto al material faunístico, únicamente se recuperaron algunos fragmentos de cascara de huevo de Rheidae y una placa dérmica de Dasipodidae. También se halló en este sitio una semilla carbonizada de Prosopis sp., la cual fue datada como moderna.

\section{Discusión y conclusiones}

En el comienzo del presente trabajo planteamos que el modelo biogeográfico más utilizado para explicar la dinámica poblacional en el sur de Mendoza aborda el aspecto cronológico de las ocupaciones humanas y el uso de los recursos disponibles en la región, vinculado a un proceso de aumento demográfico, saturación del espacio, reducción de la movilidad e intensificación en la explotación de recursos durante el Holoceno tardío. Asimismo, planteamos un modelo alternativo en el que se sugiere que no se habría dado un proceso de intensificación que implicara reducción de la movilidad ni disminución de los recursos de mayor rendimiento económico, sino que por el contrario, habría habido un aumento en la movilidad residencial y con ello un aprovechamiento de recursos locales de cada área.

Si bien resta analizar gran parte del material lítico y cerámico, por el momento las evidencias presentadas demuestran una amplia circulación humana. Si bien todavía no podemos establecer si hubo variaciones temporales en este aspecto, o si se trata de movimientos residenciales o logísticos, tanto en el desierto Andino como en el de Patagonia, existen evidencias de obsidianas provenientes de canteras a $200 \mathrm{~km}$ de distancia. En cuanto a la tecnología cerámica, los estilos decorativos provenientes de ambas vertientes cordilleranas en los sitios del desierto Andino señalan la existencia de redes de interacción desde al menos unos 2000 años AP. Por otro lado en el desierto Patagónico la presencia de cerámica Agrelo podría estar vinculando estos grupos con poblaciones de Agricultores del Norte de la provincia de Mendoza.

En cuanto a la temporalidad de las ocupaciones humanas, se cumplen las expectativas planteadas tanto por el modelo de Neme y Gil para la macro región como lo planteado en este trabajo para la cuenca del río Diamante, ya que las primeras ocupaciones se registran en sitios de las provincias fitogeográficas de Monte (sitio El Chancho) y Patagonia (sitios Agua del Médano y El Mallín) (Figura 1). Durante el Holoceno medio, se registra una ausencia total de fechados, como se observa en otros sectores del sur de Mendoza (ver discusiones sobre este tema en Garvey 2012; Gil et al. 2005; Neme y Gil 2009; 2010) y hacia el Holoceno tardío, todas las áreas se encuentran ocupadas (Figura 2).

En cuanto al consumo de recursos y lo esperado por el modelo de intensificación propuesto para la región, la evidencia zooarqueológica no es suficiente como para realizar una comparación estadística de la riqueza y diversidad taxonómica consumida antes y después del supuesto proceso de intensificación (2000 años AP). Una de las razones de esto, es la mala preservación de los especímenes óseos que dificultan una buena identificación taxonómica de los mismos. Por otro lado, el modelo alternativo al proceso de intensificación plantea que la riqueza y diversidad de fauna consumida está en relación con la diversidad de fauna disponible en cada provincia fitogeográfica. Según los datos presentados aquí, hay una mayor cantidad de presas (NTAXA) consumidas en el desierto Altoandino, que está influida por la disponibilidad de fauna que concentran las vegas de altura y la laguna del Diamante. La riqueza (número 
de taxones consumidos) de cada sitio está en estrecha vinculación con la disponibilidad de recursos locales, aunque también puede estar relacionado con el tamaño de la muestra. El valor de NTAXA más alto se observa en LD-4, donde hay recursos acuáticos que ofrece la laguna y también un mayor número de especímenes, siguiendo en orden de importancia los sitios localizados en el desierto Patagónico (Tabla 1).

Si analizamos el tipo de recursos consumidos en cada una de las provincias fitogeográficas sin considerar la temporalidad del registro, observamos que hay tanto recursos de alto como de bajo ranking (Corbat 2016), y un predominio de consumo de fauna local en cada uno de los sitios. La excepción la constituyen los dasipódidos que se localizan en sitios de la provincia fitogeográfica Altoandina donde no se encuentran naturalmente y se estima han sido transportados desde zonas más bajas. Lo mismo sucede con un fragmento de cáscara de huevo de Rheidae hallado en el sitio LD-4, ya que esta familia no se encuentra a la altitud en la que se halla dicho sitio (Giardina 2010). Por otro lado, los índices de diversidad son concordantes con las expectativas de una mayor diversidad en lugares de mayor productividad primaria. Los sitios del desierto Patagónico poseen valores más altos de dicho índice en comparación con los sitios del desierto Altoandino (Tabla 1). Los escasos restos de fauna provenientes del sitio el Chancho del desierto de Monte, nos impiden incorporar este ambiente a las comparaciones.

En lo que respecta al registro arqueobotánico, a partir de las evidencias de los sitios, Alero Montiel, El Mallín y El Carrizalito, localizados en el desierto Patagónico, Llano et al. (2012) señalan aumento en la diversidad de especies botánicas, tanto silvestres como domesticas de menor rendimiento económico incorporadas a la dieta en los últimos 1500 años AP, lo que según los autores, es consecuencia del proceso de intensificación regional. Sin embargo, dadas las características del registro arqueobotánico utilizado para defender la hipótesis de la intensificación, consideramos que esta evidencia no es suficiente. En primer lugar no existen evidencias claras de un cambio en el consumo de estos recursos a lo largo del Holoceno tardío. La cantidad de muestras no alcanza un número mínimo que permiten establecer análisis estadísticos para determinar la significancia de un cambio de estrategias en la explotación de estos recursos. Por otro lado sólo poseemos evidencias de los últimos 1500 años y en sitios en donde las técnicas de excavación no permitieron la recuperación del registro en su totalidad.

De los dos modelos alternativos propuestos para el área de estudio, que en términos sencillos podríamos confrontar como modelo de intensificación en el que los cambios demográficos implicaron intensificación producto de la saturación del espacio versus el modelo alternativo en el que los cambios demográficos no implicaron saturación del espacio ni intensificación, consideramos que las evidencias presentadas en este trabajo apuntan hacia la segunda alternativa.

Por el momento, las evidencias presentadas no indican una saturación del espacio ni disminución de los mejores recursos, y la variabilidad de los recursos explotados responden a la variabilidad que ofrece cada región, en lugar de un uso de recursos de menor rendimiento económico. Por otra parte, la presencia de cerámica, obsidiana, plantas domésticas y fauna no local en todos los pisos altitudinales, indicaría una alta movilidad de las personas que no necesariamente implican mayor territorialidad como plantea el modelo de saturación del espacio, en donde se esperarían indicadores de territorialidad como indicadores étnicos como estilos cerámicos predominantes en todos los sitios o violencia interpersonal. Sin bien aún resta evaluar en profundidad ambas alternativas, mientras no hayan evidencias claras de depresión de recursos (disminución en la cantidad de los recursos mejor renqueados) ni de territorialidad creemos que la explicación más parsimoniosa es la de un aumento en los rangos de acción producto de la mayor movilidad residencial.

A partir del presente trabajo se ha planteado el estado de la cuestión en la cuenca del río Diamante y se han generado nuevas expectativas con las cuales contrastar dos modelos alternativos. Para poder avanzar con la contrastación de hipótesis de ambos modelos, se está desarrollando un proyecto de investigación que incluye muestreos de gran escala bajo un enfoque estadístico distribucional en el que abordarán los tres desiertos con la misma intensidad (Franchetti et al. 2016). Asimismo se plantea la realización de nuevas excavaciones para corregir los sesgos cronológicos y contextuales que encontramos en el registro arqueológico de las primeras excavaciones realizadas en el área.

\section{Agradecimientos}

Queremos agradecer especialmente a la familia Gil, quienes nos reciben cálidamente en sus casas cada año durante nuestros trabajos de campo. Por otro lado, agradecemos el tiempo y el trabajo de los evaluadores del presente artículo, quienes comentaron y corrigieron versiones previas del mismo. Parte de los trabajos de campo fueron realizados con subsidios de ANPCyT (PICT2012-1015 у PICT 2015 3707).

\section{REFERENCIAS BIBLIOGRÁFICAS}

Andreoni, D. 2014. Plantas leñosas y estrategias humanas en el Sur de Mendoza: una aproximación arqueobotánica. Tesis doctoral inédita, Facultad de Ciencias Naturales y Museo, Universidad Nacional de La Plata, La Plata.

Bettinger, R. 1991. Aboriginal Occupation at High Altitude: Alpine Villages in the White Mountains of Eastern California. American Anthropologist, 93: 657-679. 
Bird, D. y J. O'Connell. 2006. Behavioral Ecology and Archaeology. Journal of ArchaeologicalResearch14: 143-188.

Böcher, T., J. Hjerting, \& K., Rahn 1972. Botanical Studies in the Atuel Valley Area, Mendoza Province, Argentina. Parte I, II, III. Dansk Botansk Arkiv, Bind 22, N³. Kobenhavn.

Borrero, L. 1989-1990. Evolución cultural divergente en la Patagonia austral. Anales del Instituto de la Patagonia; Serie Ciencias Sociales 19: 133-140. Punta Arenas, Chile.

Borrero, L. 2012. La Patagonia Cuyana, Logros y Desafíos. Neme, G. y A. Gil (editores) en Paleoecología Humana en el sur de Mendoza, pp. 281-295. Publicaciones de la Sociedad Argentina de Antropología. Buenos Aires.

Cabrera, A. 1971. Fitogeografía de la República Argentina. Boletín de la Sociedad Argentina de Botánica: 14: 1-42.

Cabrera, A. 1976. Regiones fitogeográficas argentinas. En: Enciclopedia Argentina de Agricultura y Jardinería; Tomo 2. Ediciones ACME. Buenos Aires

Corbat, M. 2016. Variabilidad ambiental y sociocultural en la explotación de peces en el centro-occidente argentino: una evaluación zooarqueológica. Tesis doctoral inédita. Universidad de Buenos Aires, Facultad de Filosofía y Letras

Cortegoso, V.; M. Glascock; A. De Francesco; V. Durán; G. Neme; A. Gil; M. Giesso; L. Sanhueza; L. Cornejo; R. Barberena \& M. Bocci. 2014. Chemical characterization of obsidian in central western Argentina and central Chile: archaeological problems and perspectives. Physical, chemical and biological markers in argentine archaeology: theory, methods and applications. Débora M. Kligmann y Marcelo R. Morales (Eds.). BAR International Series, Archaeopress, Oxford.

Cortegoso, V.; R. Barberena; V. Durán \& G. Lucero. 2016. Geographic vectors of human mobility in the Andes (34-36 $\mathrm{S}$ ): Comparative analysis of 'minor' obsidian sources. Quaternary International. http://dx.doi.org/10.1016/j.quaint.2015.11.133

De Francesco, A; V. Durán; A. Bloise \& G. Neme. 2006. Caracterización y procedencia de obsidianas de sitios arqueológicos del área natural protegida Laguna del Diamante (Mendoza, Argentina) con metodología no destructiva por fluorescencia de rayos (XRF). Durán V. y V. Cortegoso (Eds.), En Arqueología y Ambiente de Áreas Naturales Protegidas de la Provincia de Mendoza (pp. 53-67). Anales de Arqueología y Etnología, 61.

Durán, V.; M. Giesso; M. Glascock; G. Neme; A. Gil \& L. Sanhueza. 2004. Estudio de fuentes de aprovisionamiento y redes de distribución de obsidiana durante el Holoceno Tardío en el sur de Mendoza (Argentina). Estudios Atacameños 28: 25-43.

Durán, V.; G. Neme; V. Cortegoso \& A. Gil.2006. Arqueología del Área Natural Protegida Laguna del Diamante (Mendoza, Argentina). Editores: Durán V. y V. Cortegoso, En Arqueología y Ambiente de Áreas Naturales Protegidas de la Provincia de Mendoza, pp.: 81-134. Volumen Especial N61. Anales de Arqueología y Etnología. Universidad Nacional de Cuyo, Mendoza.

Durán, V.; A. De Francesco; V. Cortegoso; G. Neme; L. Cornejo \& M. Bocci 2012.Caracterización y procedencia de obsidianas de sitios arqueológicos del Centro Oeste de Argentina y Centro de Chile con metodología no destructiva por fluorescencia de Rayos X (XRF). Intersecciones en Antropología 13: 423-437.

Franchetti, F., C. Otaola \& M. Giardina. 2016. Human Biogeography in the Diamante Valley, (Central Western Argentina): Integrating Different Data in a New Research Design. Trabajo presentado en el $81^{\circ}$ Meeting of the Society for American Archaeology. Orlando, Estados Unidos.

Gambier, M. 1979. Arqueología y paleoclima en los Andes Centrales Argentino Chilenos. Publicaciones 6: 1-10. Instituto de Investigaciones Arqueológicas y Museo, San Juan.

Gambier, M.1985. La Cultura de los Morrillos, Instituto de Investigaciones Arqueológicas y Museo, FFHyA, UNSJ, San Juan.

Garvey, R., 2012. Human Behavioral Responses to Mid Holocene Climate Changes in Northern Argentine Patagonia. Tesis doctoral inédita. University of California, Davis.

Giardina, M. 2010. El aprovechamiento de la avifauna entre las sociedades cazadoras recolectoras del sur de Mendoza: un enfoque arqueozoológico. Tesis doctoral, Facultad de Ciencias Naturales y Museo, Universidad Nacional de la Plata.

Giesso, M.; V. Durán; G. Neme; M. Glascock; V. Cortegoso; A. Gil y L. Sanhueza.2011. Application of a Portable XRF to Study Obsidian Use in the Central Andes region of Argentina and Chile. Archaeometry53 (1): 1-21.

Gil, A. 2003. Zea Mays on South American Periphery: Chronology and Dietary Importance. CurrentAnthropology44: 295-300

Gil, A. 2006. Arqueología de La Payunia (Mendoza, Argentina): el poblamiento humano en los márgenes de la agricultura. BAR International Series 1477, Archaeopress, Oxford.

Gil, A.; M. Zarate; y G. Neme. 2005. Mid-Holocene paleoenvironments and human occupation in southern South America. Quaternary International 132, 1-5.

Gonzalez Díaz, E. 1972. Descripción geológica de la Hoja 27d San Rafael, Provincia de Mendoza. Servicio Nacional Minero Geológico, Boletín 132: 1-127, Buenos Aires.

Hernández, A. 2002. Paleoetnobotánica en el Sur de Mendoza. Editores; Gil, A. y G. Neme. En: Entre Montañas y Desiertos Arqueología del sur de Mendoza. 157-180. Sociedad Argentina de Antropología: Buenos Aires

Kelly, R.1995. The foraging spectrum. Diversity in hunter gatherers life ways. Smithsonian Insttitution Press.

Lagiglia, H. 1968. Secuencias culturales del Centro Oeste Argentino: Valles del Atuel y Diamante. Revista Científica de Investigaciones 1 (4): 159- 174

Lagiglia, H. 1977. Arqueología y ambiente natural de los valles del Atuel y del Diamante. Tesis doctoral, Facultad de Ciencias Naturales y Museo, Universidad Nacional de la Plata.

Llano C. L 2012. Aprovechamiento de los Recursos vegetales entre las sociedades cazadoras-recolectores del Sur de Mendoza. Tesis Doctoral inédita, Universidad Nacional del Comahue. 
Llano, C., G. Neme \& C. Michieli. 2012. Plant use intensification among hunter-gatherers in the Diamante river basin, Argentina. Before Farming 2: 1-15.

Llano, C. \& V. Durán. 2014. The introduction of wheat in Mendoza, Argentina during the sixteenth century AD.: Archaeobotanical evidence. Latin American Antiquity 25: 462 - 472.

Mares, M., J. Morello \& G. Goldstein. 1985. The Monte desert and other subtropical semi-arid biomes of Argentina, with comments on their relation to North American arid areas. Editores; $\mathrm{M}$. Evenari; I. Noy-Meir y D. Goodall. En Hot Deserts and Arid Shrublands. Pp.203-237. Amsterdam: Elsevier Science.

McCain, C. \& J. Grytnes. 2010. Elevational Gradients in Species Richness. In: Encyclopedia of Life Sciences (ELS). John Wiley \& Sons, Ltd: Chichester DOI: 10.1002/9780470015902.a0022548

Muiño, W., A. Prina \& G. Alfonso. 2012. Flora altoandina de la Reserva Laguna del Diamante (Mendoza, Argentina). Chloris Chilensis. Revista chilena de flora y vegetacion. 15: 1-89

Neme, G. 2002. Arqueología del Alto Valle del Río Atuel: Modelos, Problemas y Perspectivas en el Estudio de las Regiones de Altura del Sur de Mendoza. Editores; Gil, A. y G. Neme. En: Entre Montañas y Desiertos: Arqueología del Sur de Mendoza: 65-83. Sociedad Argentina de Antropología, Buenos Aires.

Neme, G. 2007. Cazadores-Recolectores de Altura en los Andes Meridionales. Oxford: BAR International Series 1591.

Neme, G. 2016. El Indígeno and high-altitude human occupation in the southern Andes. Latin American Antiquity. En prensa.

Neme, G. \& A. Gil. 2008a. Biogeografía humana en los Andes meridionales: tendencias arqueológicas en el sur de Mendoza. Chungará 40 (1): 5-18.

Neme, G. \& A. Gil. 2008b. Faunal Exploitation and Agricultural Transitions in the South American Agricultural Limit. International Journal of Osteoarchaeology18: 293-306.

Neme, G. \& A. Gil. 2009. Human Occupation and Increasing Mid-Holocene Aridity Southern. Current Anthropology 50 (1), 149-163.

Neme, G. \& A. Gil 2010. What Happened during the MidHolocene in Arid Western Argentina? A Reply to García. Current Anthropology 51 (3): 417-419.

Neme, G.; F. Bonat; A. Gil; M. Zarate; A. Tripaldi \& G. Brook 2009. Procesos de formación de sitios y ocupación humana en los campos de médanos de la planicie mendocina. El sitio el chancho--1 y sus implicancias para el poblamiento temprano del oeste argentino. Trabajo presentado en el XVIII Congreso Nacional de Arqueología Chilena.

Neme, G.; A. Gil; C. Otaola \& M. Giardina. 2015. Resource Exploitation and Human Mobility: Trends in the Archaeofaunal and Isotopic Record from Central Western Argentina. International Journal of Osteoarchaeology. DOI: 10.1002/oa.2359.

Neme, G.; C. Morgan; N. Sugrañez; L. Salgán; A. Gil; C. Otaola; M. Giardina \& C. Llano. 2016. Risco de los Indios: Ocupaciones humanas de altura en la Cuenca del río Diamante. Relaciones de la Sociedad Argentina de Antropología. En Revisión

Otaola, C. 2013. Zooarqueología y Tafonomía en la Cordillera de los Andes. Tesis doctoral, Universidad de Buenos Aires.

Pianka, E. 1983. Ecología Evolutiva. Ediciones Omega S.A. Barcelona.

Planella, M. \& M Tagle. 2004. Inicios de presencia de cultígenos en la Zona Central de Chile, períodos Arcaico y Agroalfarero Temprano. Chungara, volumen especial: 387-400.

Polanski, J. 1963. Estratigrafía, Neotectónica y Geomorfología del Pleistoceno entre los ríos Diamante y Mendoza. Revista de la Asociación Geológica Argentina. 17: (3-4): 128-349.

Rahbek, C. 1995. The Elevational Gradient of Species Richness: A Uniform Pattern? Source: Ecography, 18 (2): 200-205.

Roig, F.A., 1972. Bosquejo fisionómico de la vegetación de la provincia de Mendoza. Boletín de la Sociedad Argentina de Botánica: 49-80.

Roig, V.G. 1972. Esbozo general del poblamiento animal en la provincia de Mendoza. Boletín de la Sociedad Argentina de Botánica: 81-88. .

Roig, F.; E. Martínez Carretero \& E. Méndez. 2000. Mapa de vegetación de la Provincia de Mendoza, Editores Abraham M. y F. Rodríguez Martínez, En: Recursos y problemas ambientales de zona árida. pp. 10. Mendoza.

Roig, F.; S. Roig-Junenty V. Corbalán. 2009. Biogeography of the Monte Desert F.A. Journal of Arid Environments 73: 164-172.

Rusconi, C. 1962. Poblaciones Pre y Posthispánica de Mendoza. Tomo 3, Arqueología. Gobierno de Mendoza. Mendoza.

Sepúlveda, E.; F. Carpio; M. Regairaz; J. Zanettini \& M. Zárate. 2001. Hoja geológica 3569'll, San Rafael, provincia de Mendoza. Instituto de Geología y Recursos minerales, Servicio geológico Minero Argentino. Boletín 321. Segunda edición revisada, 59 pags. Buenos Aires

Shennan, S. 2002. Archaeology and Evoutionary Ecology. World Archaeology 34 (1): 1-5.

Sosa Morales, N. 1979. Historia de un pueblo la villa vieja. Serie Libros / Museo de Historia Natural, Dirección Municipal de Cultura, Departamento de San Rafael, Provincia de Mendoza, Argentina, no. 1,

Tripaldi, A., A. Gil, M. Zárate, G. Neme \& G. Brook 2009. Paleoambientes y ocupaciones humanas de la planicie sanrafaelina (Mendoza Argentina) durante el Pleitoceno tardíoHoloceno temprano. Trabajo presentado en el IV Congreso de Cuaternario y Geomorfología.

Videla, F.; M. Rosi; S. Puig \& M. Cona. 1998. Vertebrados de Mendoza y sus adaptaciones al ambiente árido. Editorial INCA, Mendoza.

Winterhalder, B. \& E. Smith. 1992. Evolutionary ecology and the social sciences. Editores: Smith, E. y B. Winterhalder. En Evolutionary Ecology and Human Behavior. Aldine de Gruyter, New York. 